\title{
Mathematical and Statistic Method to Diagnostic of Metastasis based on Radiology Images for Breast Cancer
}

\author{
Minoo Tabandegan, ${ }^{1, *}$ Ali Akbar Khadem, ${ }^{1}$ Neda Mirzaie, ${ }^{1}$ and Jalal Jalal Shokouhi ${ }^{2}$ \\ ${ }^{1}$ Azad University of Medical Sciences \\ ${ }^{2}$ Jaam E Jam Medical Center, Tehran, Iran \\ "Corresponding author: Minoo Tabandegan, Azad University of Medical Sciences. E-mail: m.tabandegan@gmail.com
}

Received 2016 December 21; Accepted 2017 February 08.

\begin{abstract}
One out of eight women face with the problem of breast cancer in her life. It is the second causes of women death in the world. Detection of breast cancer metastasis at the earliest stage is important to predict and control of breast cancer progression. It can spread to the different parts of the body, such as are the bone, liver, and lung and etc. How and where does the breast metastasis grow and migrate may open many questions to the radiologist and medical Scientifics. In this study, we discussed about the possibility of the breast metastasis to the other parts of the body and using statistic method we are able to estimate the frequency of metastasis from breast to the other parts. We have selected statistic method to this research and we studied over 200 patients (effected by breast cancer) using radiology image data. We save the history of the patient and gather all patient data in a table. Result and discussion: the result show that the most metastasis of breast occur in the brain and the less occur in liver. The result may help radiologist to understand better origin of metastasis and in case the possibility is high, the radiologist may ask for breast imaging. A New discussion comes to the mind that, do we need to perform breast imaging if radiologist faced with metastasis on brain?
\end{abstract}

This is an abstract presented in the 33rd Iranian congress of radiology (ICR) and the 15th congress of Iranian radiographic science association (IRSA). 